\title{
HYPERFINE INTERACTIONS OF THE NUCLEAR SPIN WITH ELECTRON SYSTEMS IN THE DENSITY MATRIX METHODS
}

\author{
R. L. Pavlov ${ }^{1}$, P. P. Raychev ${ }^{1}$, M. Dimitrova-Ivanovich ${ }^{2}$, C. Valdemoro ${ }^{3}$ \\ ${ }^{1}$ Institute for Nuclear Research and Nuclear Energy, Bulgarian Academy of Sciences, \\ 72 Tsarigradsko Chaussee, BG-1784 Sofia, Bulgaria, \\ ${ }^{2}$ Institute of Solid State Physics, Bulgarian Academy of Sciences, \\ 72 Tsarigradsko Chaussee, BG-1784 Sofia, Bulgaria, \\ ${ }^{3}$ Instituto de Matematicas y Fisica Fundamental, CSIC, 123 Serrano, 28006 Madrid, Spain
}

(Received October 1, 2001)

\begin{abstract}
The matrix elements and expectation values of the operators of the hyperfine electron-nuclear interactions in many-electron systems are presented in an analytical form, which can be applied for numerical calculations in the density matrix methods. The electron-nuclear spin-spin and contact interactions are considered, as well as the interaction between nuclear-spin and electron orbital motions. These interactions which take part in the effective Breit-Pauli Hamiltonian, determine hyperfine structure of the basic effects in the spectroscopy, which can be observed for example in electron spin resonance (ESR) and nuclear magnetic resonance (NMR) experiments. Applying the Wigner-Eckart theorem, using the irreducible tensor-operators technique and the spin-space separation scheme, the matrix elements and expectation values of these relativistic corrections are expressed in a form suitable for direct numeric calculations. The final result is presented as a product (or sums of products) of factors determined by the spin and (or) the angular momentum symmetry, and a spatial part determined by the action of the symmetrized tensor-operators on the normalized matrix or function of the spin distribution or the matrix of the charge distribution. The action of these space tensor operators is the same for a given spin multiplet. This action can be expressed by a standard procedure, well defined in the theory of the irreducible tensor-operators.

Key words: electron-nuclear interaction, hyperfine structure, relativistic correction, density matrix, Clebsch-Gordon coefficients.
\end{abstract}

PACS number(s): 31.20.Sy, 31.30.Gs, 31.30.Jv

\section{INTRODUCTION}

In the formalism of the reduced density matrices and functions (RDM \& RDF)[1-3], the matrix elements, as well as expectation values of the different types of spininvolving operators take the form of a product of space and spin factors [1-4]. The spin part is determined by the spin symmetry and is reduced to $3 j$-symbols, while the spatial part is determined by the action of space operators on the spin distribution or spin correlations matrices or functions $[1,4,5]$. The spin distribution and spin correlations matrices and functions are built from the spatial parts of the RDMs of first and second order, respectively. In this approach, the space-spin separation results, first, from the possibility to separate the space and spin variables in RDMs [6-9] and, second, from the use of the split RDMs in order to represent the matrix elements of the perturbation terms in the Hamiltonian $[1,4,10]$.

In terms of the RDMs and RDFs and in the scheme of the space-spin separation, the matrix elements and expectation values of the various spin-involving operators (presented in Refs. [1,4]) are connected with the relativistic corrections in the Breit-Pauli Hamiltonian [1-5]. The matrix elements and expectation values of the operators corresponding to the different types of relativistic corrections are reduced to the products of a factor determined by the spin symmetry which characterize a spin state or a transition between two states in a given spin multiplet, and a space part which is independent of the spin state or of a transition amplitude between two states. This space part is expressed by the action of the space operator, considered in general form, on the space part of the relevant spin distribution, or spin correlations matrices or functions.

In Refs. [11,12] we have taken a step further in the treatment of the matrix elements and expectation values of one of the main relativistic corrections, namely, the spin-orbit interactions and spin-spin, electron-electron interactions terms, in a form convenient for numerical calculations in the density matrix methods.

In the present paper we shall consider the matrix elements and expectation values of another type of spininvolving relativistic corrections, namely the electronnuclear spin-spin (dipolar) and electron-nuclear contact interactions, as well as the interaction between nuclearspin and electron orbital motion. These interactions terms in the effective Breit-Pauli Hamiltonian determine the hyperfine structure of the basic effects in the spectroscopy, observed for example in the EPR and NMR experiments.

Using the technique of the irreducible tensor operators and applying the Wigner-Eckart theorem, the matrix elements and expectation values of these relativistic corrections are expressed as products, or sums of products, of factors, determined by the spin symmetry and (or) the orbital momentum symmetry, and a spatial part deter- 
mined by the action of the symmetrized tensor-operators over the normalized matrix or function of the spin distribution or the matrix of the charge distribution. The action of these space tensor operators is the same for a given spin multiplet and is independent of the investigated (splitting or transition) effect. This action is reduced to a standard procedure, well defined in the theory of the irreducible tensor-operators. The expectation values of these relativistic corrections, which give the value of splitting of the energy levels, are expressed in an analytical form suitable for numerical calculations. We also consider the transition matrix elements of these operators, which give the contribution of the electron-nuclear interactions under consideration to the corresponding induced transitions.

\section{SPIN DISTRIBUTION MATRICES AND DENSITIES}

\section{A. Matrix elements in the RDM formalism}

The RDM of the order $s$ ( $s$-RDM) of an $N$-electron system $(1 \leq s \leq N)$ in the state $K$, described by a wave function $\Psi_{K}\left(\tau_{1}, \ldots, \tau_{N}\right)$, which is eigenfunction of the operators $S^{2}$ and $S_{Z}$, has the form $[1-3,13-16]$

$$
\begin{aligned}
\rho\left(K K \mid \tau_{1}, \ldots, \tau_{s} ; \tau_{1}^{\prime}, \ldots, \tau_{s}^{\prime}\right) & =N(N-1) \ldots(N-s+1) \\
& \times \int \Psi_{K}\left(\tau_{1}, \ldots, \tau_{s} ; \tau_{s+1}, \ldots, \tau_{N}\right) \Psi_{K}^{*}\left(\tau_{1}^{\prime}, \ldots, \tau_{s}^{\prime} ; \tau_{s+1}, \ldots, \tau_{N}\right) d \tau_{s+1} \ldots d \tau_{N}
\end{aligned}
$$

where $\tau_{i}=\left(\mathbf{r}_{i}, \sigma_{i}\right), \mathbf{r}_{i}$ is the position vector of the $i$-th electron and $\sigma_{i}$ is the spin variable.

The corresponding RDF of the order $s(s-\mathrm{RDF})$ is defined by the expression

$$
\rho\left(K K \mid \tau_{1}, \ldots, \tau_{s}\right)=\left.\rho\left(K K \mid \tau_{1}, \ldots, \tau_{s} ; \tau_{1}^{\prime}, \ldots, \tau_{s}^{\prime}\right)\right|_{\tau_{\{i\}}^{\prime}=\tau_{\{i\}}}=\rho\left(K K \mid \tau_{1}, \ldots, \tau_{s} ; \tau_{1}, \ldots, \tau_{s}\right)
$$

The generalized transition $s$-RDM between the states $K$ and $K^{\prime}$ described by $\Psi_{K}$ and $\Psi_{K^{\prime}}$ has the form [1-3]:

$$
\begin{aligned}
\rho\left(K K^{\prime} \mid \tau_{1}, \ldots, \tau_{s} ; \tau_{1}^{\prime}, \ldots, \tau_{s}^{\prime}\right) & =N(N-1) \ldots(N-s+1) \\
& \times \int \Psi_{K}\left(\tau_{1}, \ldots, \tau_{s} ; \tau_{s+1}, \ldots, \tau_{N}\right) \Psi_{K^{\prime}}^{*}\left(\tau_{1}^{\prime}, \ldots, \tau_{s}^{\prime} ; \tau_{s+1}, \ldots, \tau_{N}\right) d \tau_{s+1} \ldots d \tau_{N}
\end{aligned}
$$

For the transition $s$-RDF we have a similar expression which follows from Eq. (2).

The expectation value of a $s$-particle operator, $\mathbf{F}\left(i_{1}, i_{2}, \ldots i_{s}\right)$, can be written as

$$
\begin{aligned}
\left\langle\mathbf{F}\left(i_{1}, i_{2}, \ldots i_{s}\right)\right\rangle & =\operatorname{Sp}_{i_{1}, i_{2}, \ldots i_{s}} \mathbf{F}\left(i_{1}, i_{2}, \ldots i_{s}\right) \rho\left(\tau_{1}, \ldots, \tau_{N} ; \tau_{1}^{\prime}, \ldots, \tau_{N}^{\prime}\right) \\
& =\operatorname{Sp}_{i_{1}, i_{2}, \ldots i_{s}} \mathbf{F}\left(i_{1}, i_{2}, \ldots i_{s}\right) \rho\left(\tau_{1}, \ldots, \tau_{s} ; \tau_{1}^{\prime}, \ldots, \tau_{s}^{\prime}\right)
\end{aligned}
$$

In expression (4) $\mathrm{Sp}_{i_{1}, i_{2}, \ldots i_{s}}$ denotes the operator of the integration over particle coordinates labeled with the corresponding numbers, after identification of the primed and unprimed coordinates.

In many-electron theory, the expectation values and matrix elements of symmetric sums of identical operators are of major importance. Then, for the expectation values of a symmetrized sum of identical s-particle operators, $\mathbf{F}\left(i_{1}, i_{2}, \ldots i_{s}\right)$, (assuming that the numbers are ordered as follow $i_{k}, 1 \leq i_{1}<i_{2}<\ldots<i_{s} \leq N$ ), we obtain

$$
\begin{aligned}
\left\langle\sum_{\left\{i_{s}\right\}} \mathbf{F}\left(i_{1}, i_{2}, \ldots i_{s}\right)\right\rangle & =\left\langle\Psi_{K}\left(\tau_{1}, \ldots, \tau_{N}\right)\left|\sum_{\left\{i_{s}\right\}} \mathbf{F}\left(i_{1}, i_{2}, \ldots i_{s}\right)\right| \Psi_{K}\left(\tau_{1}, \ldots, \tau_{N}\right)\right\rangle \\
& =\frac{1}{s !} \operatorname{Sp} \mathbf{F}\left(i_{1}, i_{2}, \ldots i_{s}\right) \rho\left(K \mid \tau_{i_{1}}, \ldots, \tau_{i_{s}} ; \tau_{i_{1}}^{\prime}, \ldots, \tau_{i_{s}}^{\prime}\right)
\end{aligned}
$$

where $\sum_{\left\{i_{s}\right\}}$ means a summation over all possible sets $\left(i_{1}, i_{2} \ldots, i_{N}\right)$. Usually, only RDMs or RDFs of 1 st or 2nd order are of importance. Higher-order matrices or functions are used only in specific cases.

After separation of the space and spin variables $[1,2,6]$, the 1-RDM takes the form 


\section{R. L. PAVLOV, P. P. RAYCHEV, M. DIMITROVA-IVANOVICH, C. VALDEMORO}

$$
\rho\left(\tau_{1} ; \tau_{1}^{\prime}\right)=\sum_{\gamma, \gamma^{\prime}=\alpha, \beta} \rho^{\gamma, \gamma^{\prime}}\left(\mathbf{r}_{1} ; \mathbf{r}_{1}^{\prime}\right) \gamma\left(\sigma_{1}\right) \gamma^{\prime *}\left(\sigma_{1}^{\prime}\right),
$$

where the $\rho^{\gamma, \gamma^{\prime}}\left(\mathbf{r}_{1} ; \mathbf{r}_{1}^{\prime}\right)$ are the space components and the $\gamma(\sigma) \quad(\gamma=\alpha, \beta)$ are two spin one-electron wave functions. The space components form the charge and spin distribution matrices (section II.B):

$$
\rho\left(\mathbf{r}_{1} ; \mathbf{r}_{1}^{\prime}\right)=\rho^{\alpha, \alpha}\left(\mathbf{r}_{1} ; \mathbf{r}_{1}^{\prime}\right)+\rho^{\beta, \beta}\left(\mathbf{r}_{1} ; \mathbf{r}_{1}^{\prime}\right) .
$$

For the expectation values of a sum of identical oneparticle operators, making use of Eq. (5) we obtain

$$
\begin{aligned}
& \left\langle\sum_{i} \mathbf{F}(i)\right\rangle=\left\langle\Psi_{K}\left(\tau_{1}, \ldots, \tau_{N}\right)\left|\sum_{i} \mathbf{F}(i)\right| \Psi_{K}\left(\tau_{1}, \ldots, \tau_{N}\right)\right\rangle \\
& =\operatorname{Sp} \mathbf{F}(1) \rho\left(K \mid \tau_{1} ; \tau_{1}^{\prime}\right)=\int_{\tau_{1}^{\prime}=\tau_{1}} \mathbf{F}(1) \rho\left(K \mid \tau_{1} ; \tau_{1}^{\prime}\right) d \tau_{1}
\end{aligned}
$$

\section{B. Spin distribution matrices and functions}

In the spin eigenstate (with the eigenvalues $S(S+1)$ and $M$ of the operators $S^{2}$ and $S_{z}$, respectively ) the spin distribution matrix can be written down in the following form $[1,2,4,5]$

$$
q\left(\mathbf{r}_{1} ; \mathbf{r}_{1}^{\prime}\right)=1 / 2\left[\rho^{\alpha, \alpha}\left(\mathbf{r}_{1} ; \mathbf{r}_{1}^{\prime}\right)-\rho^{\beta, \beta}\left(\mathbf{r}_{1} ; \mathbf{r}_{1}^{\prime}\right)\right]
$$

The spin distribution matrices $q^{(M)}\left(\mathbf{r}_{1} ; \mathbf{r}_{1}^{\prime}\right)$ for the different spin eigenstates (with $M=S, S-1, \ldots,-S$ ) can be expressed in terms of the normalized spin distribution matrices $D_{S}\left(\mathbf{r}_{1} ; \mathbf{r}_{1}^{\prime}\right)$, which are independent of $M[17,18]$ :

$$
\begin{aligned}
q^{(M)}\left(\mathbf{r}_{1} ; \mathbf{r}_{1}^{\prime}\right) & \equiv q\left(K K \mid \mathbf{r}_{1} ; \mathbf{r}_{1}^{\prime}\right)=\frac{M}{S} q\left(\bar{K} \bar{K} \mid \mathbf{r}_{1} ; \mathbf{r}_{1}^{\prime}\right) \\
& =M D_{S}\left(\mathbf{r}_{1} ; \mathbf{r}_{1}^{\prime}\right)
\end{aligned}
$$

where:

$$
D_{S}\left(\mathbf{r}_{1} ; \mathbf{r}_{1}^{\prime}\right)=\frac{1}{S} q\left(\bar{K} \bar{K} \mid \mathbf{r}_{1} ; \mathbf{r}_{1}^{\prime}\right)
$$

Here $K$ is the index of the spin state corresponding to $\left\langle S_{z}\right\rangle=M$ and $\bar{K}$ is the index corresponding to the maximal value $M=S$ and $q\left(K K \mid \mathbf{r}_{1} ; \mathbf{r}_{1}^{\prime}\right)$ is presented in form (9), using the general definition $[1,4]$

$$
q\left(K K^{\prime} \mid \mathbf{r}_{1} ; \mathbf{r}_{1}^{\prime}\right)_{m}^{1}=\int_{\sigma_{1}^{\prime}=\sigma_{1}} S_{m}^{1}(1) \rho\left(K K^{\prime} \mid \tau_{1} ; \tau_{1}^{\prime}\right) d \sigma_{1},
$$

where $S_{m}^{1}(i), m=0, \pm 1$ are symmetrized components of the spin moment $\mathbf{S}(i)$ of the electron with the number $i$.
Similar formulas can be written for the spin distribution functions when $\mathbf{r}_{1}=\mathbf{r}_{1}^{\prime}$.

The matrices of the spin distribution for the transition between the states $K\left(S M_{S}\right)$ and $K^{\prime}\left(S^{\prime} M_{S^{\prime}}\right)$ satisfy relations $[1,4]$

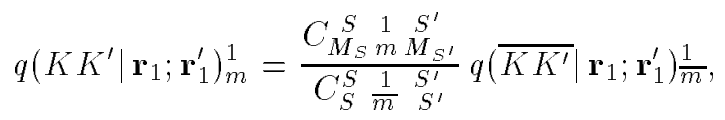

where $C_{M_{S}}^{S} \frac{1}{M_{S}} \stackrel{S}{\prime}_{M}$ are the Clebsch-Gordon coefficients. From their properties it follows that $m=M_{S^{\prime}}-M_{S}$ and $\bar{m}=S^{\prime}-S$. By analogy with (12) one obtain

$$
q\left(\overline{K K^{\prime}} \mid \mathbf{r}_{1} ; \mathbf{r}_{1}^{\prime}\right)_{m}^{1}=\int_{\sigma_{1}^{\prime}=\sigma_{1}} S_{m}^{1}(1) \rho\left(\overline{K K^{\prime}} \mid \tau_{1} ; \tau_{1}^{\prime}\right) d \sigma_{1} .
$$

Applying the Wigner-Eckart theorem, from (13) to the transition between the states $K\left(S M_{S}\right)$ and $K^{\prime}\left(S M_{S}^{\prime}\right)$ one obtains

$$
\begin{aligned}
q\left(K K^{\prime} \mid \mathbf{r}_{1} ; \mathbf{r}_{1}^{\prime}\right)_{m}^{1} & =\frac{C_{M_{S} m \stackrel{S}{S} M_{S}^{\prime}}^{S}}{C_{S}^{S} 1 S S}\left(\overline{K K^{\prime}} \mid \mathbf{r}_{1} ; \mathbf{r}_{1}^{\prime}\right) \\
& =\left\langle S M_{S}\left|S_{m}^{1}\right| S M_{S}^{\prime}\right\rangle D_{S}\left(\mathbf{r}_{1} ; \mathbf{r}_{1}^{\prime}\right),
\end{aligned}
$$

where $S_{m}^{1}, m=0, \pm 1$ are the components of the symmetrized operator of the total spin. Eqs. (13) and (14) are generalization of Eq. (10) for the case of a transition between the different spin states.

\section{ELECTRON-NUCLEAR DIPOLAR INTERACTIONS}

The operator of dipole coupling between one electron spin magnetic moment $\mathbf{S}(i)$ and the magnetic moment $\mathbf{I}(\lambda)$ of the nucleus $\lambda$ can be written down in as in $[1,4,5]$ which separates electron and nucleus variables:

$$
\begin{aligned}
\hat{\mathbf{H}}_{e n}^{S S}= & g_{0} \alpha \alpha_{p} \sum_{\lambda, i} g_{\lambda} \mathbf{S}(i) \cdot\left[3 \frac{\mathbf{r}_{\lambda i} \otimes \mathbf{r}_{\lambda i}}{r_{\lambda i}^{5}}-\frac{\mathbf{n}^{2}(i)}{r_{\lambda i}^{3}}\right] \cdot \mathbf{I}(\lambda) \\
= & g_{0} \alpha \alpha_{p} \sum_{\lambda, i} g_{\lambda}\left\{3\left[\mathbf{S}(i) \cdot \mathbf{r}_{\lambda i}\right]\left[\mathbf{I}(\lambda) \cdot \mathbf{r}_{\lambda i}\right]\right. \\
& \left.-r_{\lambda i}^{2} \mathbf{S}(i) \cdot \mathbf{I}(\lambda)\right\} r_{\lambda i}^{-5}
\end{aligned}
$$

Here $\alpha=1 / 2 c$ is the fine structure constant, $\alpha_{p}$ is the structure constant of the proton, $g_{0}$ is the $g$-factor for the free electron [19], $g_{\lambda}$ is the $g$-factor for the nucleus $\lambda$ and $\mathbf{n}^{2}(i)$ is the unit tensor formed from the vector $\mathbf{n}(i)=\mathbf{r}_{\lambda i} / r_{\lambda i}$. We denote $\mathbf{r}_{\lambda i}=\mathbf{r}_{i}-\mathbf{R}_{\lambda}$, where $\mathbf{R}_{\lambda}$ is the position vector of the nucleus with number $\lambda$. 
The interactions, described by this operator, determine the hyperfine structure of the effects observed in the EPR experiments.

For any one nucleus $\lambda$, the sum of one electron operator in Eq. (15) can be written as

$$
\sum_{i}^{\lambda} \hat{\mathbf{H}}_{e n}^{S S}(i)=g_{0} \alpha g_{\lambda} \alpha_{p} \sum_{i} r_{\lambda i}^{-3} \mathbf{K}(i) \cdot \mathbf{I}(\lambda)
$$

where

$$
\mathbf{K}(i)=3[\mathbf{S}(i) \cdot \mathbf{n}(i)] \mathbf{n}(i)-\mathbf{S}(i) .
$$

The components of the axial vector $\mathbf{K}(i)$ can be presented in the form

$$
K_{k}(i)=\sum_{l} D_{k l} S_{l}^{(1)}(i)
$$

where

$$
D_{k l}=3 n_{k}(i) n_{l}(i)-\delta_{k l} n_{k}(i) n_{l}(i)
$$

is a traceless symmetric second-rank tensor which is proportional to the normalized spherical functions $C^{2}\left(\vartheta_{i}, \varphi_{i}\right)$ with components:

$$
C_{q}^{p}(1)=C_{q}^{p}\left(\vartheta_{1}, \varphi_{1}\right)=[4 \pi /(2 p+1)]^{1 / 2} Y_{q}^{p}\left(\vartheta_{1}, \varphi_{1}\right)
$$

As usual $Y_{q}^{p}\left(\vartheta_{1}, \varphi_{1}\right)$ are the components of the spherical functions.

The components of the symmetrized vector $\mathbf{K}^{1}(i)$ are given by

$$
K_{q}^{1}(i)=C\left[D^{2} \otimes S^{1}\right]_{q}^{1}=C \sum_{m, m^{\prime}} C_{m m^{\prime}}^{2}{ }_{q}^{1} C_{m}^{2}\left(\vartheta_{i}, \varphi_{i}\right) S_{m^{\prime}}^{1}(i)
$$

where $\left[D^{2} \otimes S^{1}\right]^{1}$ is an irreducible first-rank tensor built from the tensor product of $D^{2}$ and $S^{1}$. The constant $C$ can be derived from atomic spectroscopy theory $[20,21]$ and its value is $10^{1 / 2}$.

Using the relations given above and expressing the scalar product of $\mathbf{K}$ and $\mathbf{I}$ as a tensor product of their symmetrized forms $\mathbf{K}^{1}$ and $\mathbf{I}^{1}$, Eq. (16) takes the form

$$
\begin{aligned}
\sum_{i}^{\lambda} \hat{\mathbf{H}}_{e n}^{S S}(i) & =g_{0} \alpha g_{\lambda} \alpha_{p} \sum_{i} r_{\lambda i}^{-3} \sum_{q}(-1)^{q}(K)_{q}^{1}(i) \cdot I_{-q}^{1}(\lambda) \\
& =\sqrt{10} g_{0} \alpha g_{\lambda} \alpha_{p} \sum_{q}(-1)^{q} \sum_{m, m^{\prime}} C_{m}^{2} \frac{1}{m^{\prime}} \frac{1}{q} \sum_{i} r_{\lambda i}^{-3} C_{m}^{2}\left(\vartheta_{i}, \varphi_{i}\right) S_{m^{\prime}}^{1}(i) I_{-q}^{1}(\lambda)
\end{aligned}
$$

If one separates the electron and nuclear parts of the wavefunction, for the matrix elements of the transition between the states $K$ and $K^{\prime}$ of the electron system and $K_{\lambda}$ and $K_{\lambda}^{\prime}$ of the nucleus $\lambda$ one obtains:

$$
\begin{aligned}
& \left\langle K S M_{S} K_{\lambda}\left|\sum_{i}^{\lambda} \hat{\mathbf{H}}_{e n}^{S S}(i)\right| K^{\prime} S M_{S}^{\prime} K_{\lambda}^{\prime}\right\rangle=\sqrt{10} g_{0} \alpha g_{\lambda} \alpha_{p} \sum_{q}(-1)^{q} \sum_{m, m^{\prime}} C_{m}^{2} \frac{1}{m^{\prime}} \frac{1}{q} \\
& \times\left\langle K S M_{S}\left|\sum_{i} r_{\lambda i}^{-3} C_{m}^{2}\left(\vartheta_{i}, \varphi_{i}\right) S_{m^{\prime}}^{1}(i)\right| K^{\prime} S M_{S}^{\prime}\right\rangle\left\langle K_{\lambda}\left|I_{-q}^{1}(\lambda)\right| K_{\lambda}^{\prime}\right\rangle .
\end{aligned}
$$

We will consider only the transition between the states $K$ and $K^{\prime}$ belonging to one and the same spin multiplet. The transitions between different spin multiplets are forbidden as can be seen from the consideration of their symmetry properties.

Using Eqs. (12)-(14) we can express the matrix elements of the electron part in the following form

$$
\begin{aligned}
\left\langle K S M_{S}\left|\sum_{i} r_{\lambda i}^{-3} C_{m}^{2}\left(\vartheta i, \varphi_{i}\right) S_{m^{\prime}}^{1}(i)\right| K^{\prime} S M_{S}^{\prime}\right\rangle & =\int_{\mathbf{r}_{1}^{\prime}=\mathbf{r}_{1}} r_{\lambda 1}^{-3} C_{m}^{2}\left(\vartheta 1, \varphi_{1}\right) q\left(K K^{\prime} \mid \mathbf{r}_{1} ; \mathbf{r}_{1}^{\prime}\right)_{m^{\prime}}^{1} d \mathbf{r}_{1}^{\prime} \\
& =\left\langle S M_{S}\left|S_{m^{\prime}}^{1}\right| S M_{S}^{\prime}\right\rangle \int_{\mathbf{r}_{1}} r_{\lambda 1}^{-3} C_{m}^{2}\left(\vartheta 1, \varphi_{1}\right) D_{S}\left(\mathbf{r}_{1}\right) d \mathbf{r}_{1}
\end{aligned}
$$


where $S_{m}^{1}$ are the symmetrized components of the total spin $\mathbf{S}$ of the many electron system and $D_{S}\left(\mathbf{r}_{1}\right)=$ $q\left(\bar{K} \bar{K} \mid \mathbf{r}_{1} ; \mathbf{r}_{1}\right)_{0}^{1} / S$ is the normalized spin distribution function, i.e the spin density, which is the same within any given spin multiplet.

Applying the Wigner-Eckart theorem to the matrix element in Eq. (23) we obtain

$$
\left\langle S M_{S}\left|S_{m^{\prime}}^{1}\right| S M_{S}^{\prime}\right\rangle=\sqrt{\frac{3}{2}} \frac{1}{\sqrt{2 S+1}} C_{M_{S}}^{S} m^{\prime} M_{S}^{\prime}
$$

Finally, for the matrix elements of the operator $\hat{\mathbf{H}}_{e n}^{S S}$ for the transition between the states $K$ and $K^{\prime}$ of the electron system and $K_{\lambda}$ and $K_{\lambda}^{\prime}$ of the nuclear system one obtains

$$
\begin{aligned}
& \left\langle K S M_{S} K_{\lambda}\left|\hat{\mathbf{H}}_{e n}^{S S}\right| K^{\prime} S M_{S}^{\prime} K_{\lambda}^{\prime}\right\rangle=\sqrt{15} g_{0} \alpha \alpha_{p} \frac{1}{\sqrt{2 S+1}} \sum_{\lambda} g_{\lambda} \sum_{q}(-1)^{q} \\
& \times \sum_{m, m^{\prime}} C_{m m^{\prime}}^{2}{ }_{q}^{1} C_{M_{S}}^{S} m^{\prime} \stackrel{S}{M}_{S}^{\prime} \int r_{\lambda 1}^{-3} C_{m}^{2}\left(\vartheta_{1}, \varphi_{1}\right) D_{S}\left(\mathbf{r}_{1}\right) d \mathbf{r}_{1}\left\langle K_{\lambda}\left|I_{-q}^{1}(\lambda)\right| K_{\lambda}^{\prime}\right\rangle .
\end{aligned}
$$

In particular for a given electronic term, this matrix element takes the form

$$
\begin{aligned}
\left\langle K S M_{S} K_{\lambda}\left|\hat{\mathbf{H}}_{e n}^{S S}\right| K S M_{S} K_{\lambda}^{\prime}\right\rangle & =\sqrt{15} g_{0} \alpha \alpha_{p} \frac{1}{\sqrt{2 S+1}} \sum_{\lambda} g_{\lambda} \sum_{q}(-1)^{q} \sum_{m} C_{m}^{2}{ }_{0}^{1}{ }_{q}^{1} M_{S} \\
& \times \int r_{\lambda 1}^{-3} C_{m}^{2}\left(\vartheta i, \varphi_{i}\right) D_{S}\left(\mathbf{r}_{1}\right) d \mathbf{r}_{1}\left\langle K_{\lambda}\left|I_{-q}^{1}(\lambda)\right| K_{\lambda}^{\prime}\right\rangle .
\end{aligned}
$$

The expectation value (26) gives the width of the spectral line splitting due to the electron-nucleus spin-spin interaction.

\section{ELECTRON-NUCLEAR CONTACT INTERACTION}

The term of the electron-nuclear contact interaction in the effective Breit-Pauli Hamiltonian has the form as in $[1,4,5]$

$$
\hat{\mathbf{H}}_{e n(\mathrm{cont})}^{S S}=\frac{8}{3} \pi g_{0} \alpha \alpha_{p} \sum_{\lambda, i} g_{\lambda} \delta\left(\mathbf{r}_{\lambda i}\right) \mathbf{I}(\lambda) \cdot \mathbf{S}(i)
$$

These interactions (and the operator $\hat{\mathbf{H}}_{e n}^{S S}$ as well) determine the hyperfine structure of the effects in the EPR experiments.

Expressing the scalar product of $\mathbf{I}(\lambda)$ and $\mathbf{S}(i)$ in Eq. (27) in terms of their symmetrized components we obtain

$$
\hat{\mathbf{H}}_{e n(\mathrm{cont})}^{S S}=\frac{8}{3} \pi g_{0} \alpha \alpha_{p} \sum_{\lambda, i} g_{\lambda} \delta\left(\mathbf{r}_{\lambda i}\right) \sum_{m}(-1)^{m} S_{m}^{1}(i) I_{-m}^{1}(\lambda)
$$

Separating the wave function into the electron and nuclear part, for the matrix element of $\hat{\mathbf{H}}_{\text {en(cont) }}^{S . S}$ one easily obtains

$$
\begin{aligned}
& \left\langle K S M_{S} K_{\lambda}\left|\hat{\mathbf{H}}_{e n(\mathrm{cont})}^{S S}\right| K^{\prime} S M_{S}^{\prime} K_{\lambda}^{\prime}\right\rangle=\frac{8}{3} \pi g_{0} \alpha \alpha_{p} \sum_{\lambda} g_{\lambda} \\
& \times \sum_{m}(-1)^{m}\left\langle K S M_{S}\left|\sum_{i} \delta\left(\mathbf{r}_{\lambda i}\right) S_{m}^{1}(i)\right| K^{\prime} S M_{S}^{\prime}\right\rangle\left\langle K_{\lambda}\left|I_{-m}^{1}(\lambda)\right| K_{\lambda}^{\prime}\right\rangle
\end{aligned}
$$

Using the same procedure as in section III, for the first matrix element in this expression we have 


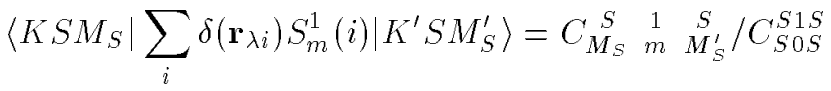

$$
\begin{aligned}
& \times \int_{\mathbf{r}_{1}^{\prime}=\mathbf{r}_{1}} \delta\left(\mathbf{r}_{\lambda 1}\right) q\left(K K^{\prime} \mid \mathbf{r}_{1} ; \mathbf{r}^{\prime}{ }_{1}\right)_{0}^{1} d \mathbf{r}_{1}=\left\langle S M_{S}\left|S_{m}^{1}\right| S M_{S}^{\prime}\right\rangle \\
& \times \int_{\mathbf{r}_{1}} \delta\left(\mathbf{r}_{\lambda 1}\right) D_{S}\left(\mathbf{r}_{1}\right) d \mathbf{r}_{1}=\sqrt{\frac{3}{2}} \frac{1}{\sqrt{2 S+1}} C_{M_{S}^{S}}^{S} \underset{m}{\stackrel{1}{M}} \stackrel{S}{M_{S}^{\prime}} D_{S}\left(\mathbf{R}_{\lambda}\right)
\end{aligned}
$$

Finally for the matrix element of $\hat{\mathbf{H}}_{e n(\text { cont })}^{S S}$, we obtain

$$
\begin{aligned}
& \left\langle K S M_{S} K_{\lambda}\left|\hat{\mathbf{H}}_{\text {en (cont) }}^{S S}\right| K^{\prime} S M_{S}^{\prime} K_{\lambda}^{\prime}\right\rangle=\frac{8}{3} \sqrt{\frac{3}{2}} \pi g_{0} \alpha \alpha_{p} \frac{1}{\sqrt{2 S+1}} \\
& \times \sum_{\lambda} g_{\lambda} \sum_{m}(-1)^{m} C_{M_{S}}^{S} \quad \stackrel{1}{m} \stackrel{S}{M_{S}^{\prime}} D_{S}\left(\mathbf{R}_{\lambda}\right)\left\langle K_{\lambda}\left|I_{-m}^{1}(\lambda)\right| K_{\lambda}^{\prime}\right\rangle .
\end{aligned}
$$

For a given term Eq. (30) formally takes the following simple form:

$$
\left\langle K S M_{S} K_{\lambda}\left|\hat{\mathbf{H}}_{e n(\mathrm{cont})}^{S S}\right| K S M_{S} K_{\lambda}^{\prime}\right\rangle \frac{8}{3} \sqrt{\frac{3}{2}} \pi g_{0} \alpha \alpha_{p} \frac{1}{\sqrt{2 S+1}} \sum_{\lambda} g_{\lambda} M_{S} D_{S}\left(\mathbf{R}_{\lambda}\right)\left\langle K_{\lambda}\left|I_{-0}^{1}(\lambda)\right| K_{\lambda}^{\prime}\right\rangle
$$

\section{NUCLEAR-ELECTRON SPIN-ORBITAL INTERACTION}

The interaction between the magnetic moment of nuclei and the orbital electronic motion which contribute to the effects of chemical shifts in NMR-experiments can be described by the operator $\hat{\mathbf{H}}_{e n}^{L S}$ in the form $[1,4,5]$ :

$$
\hat{\mathbf{H}}_{e n}^{L S}=2 \alpha \alpha_{p} \sum_{\lambda, i} g_{\lambda} r_{\lambda i}^{-3} \mathbf{I}(\lambda) \cdot \mathbf{L}_{\lambda}(i)
$$

Here, in the absence of external magnetic fields $\mathbf{L}_{\lambda}(i)$ is the angular moment of the $i$-th electron according to the nucleus $\lambda$

$$
\mathbf{L}_{\lambda}(i)=\mathbf{L}_{(i) / \lambda}=\mathbf{r}_{\lambda i} \times \mathbf{p}(i)=\mathbf{r}_{i} \times \mathbf{p}(i)-\mathbf{R}_{\lambda} \times \mathbf{p}(i) .
$$

Expressing the scalar product in (33) in terms of symmetrizied components of $\mathbf{I}(\lambda)$ and $\mathbf{L}_{\lambda}(i)$ the operator $\hat{\mathbf{H}}_{e n}^{L S}$ takes the form

$$
\hat{\mathbf{H}}_{e n}^{L S}=2 \alpha \alpha_{p} \sum_{\lambda, i} g_{\lambda} r_{\lambda i}^{-3} \sum_{m}(-1)^{m} I_{-m}^{1}(\lambda)\left(L_{\lambda}(i)\right)_{m}^{1} .
$$

In particular if one supposes that the origin of coordinate system is on the nucleus the operator (35) can be written down as

$$
{ }^{a} \hat{\mathbf{H}}_{e n}^{L S}=2 \alpha \alpha_{p} g_{\lambda} \sum_{i} r_{i}^{-3} \sum_{m}(-1)^{m} I_{-m}^{1}(\lambda) L_{m}^{1}(i)
$$

where $L_{m}^{1}(i)$ are the spherical components of the orbital angular moment of the $i$-th electron, i.e $\mathbf{L}(i)=\mathbf{r}_{i} \times \mathbf{p}(i)$. Taking into account that $\mathbf{p}(i)=-i \nabla_{i}$, and that

$$
\nabla=\mathbf{n} \frac{\partial}{\partial r}+\frac{1}{r} \nabla_{\Omega}
$$

where $\mathbf{n}$ is the normal of $\mathbf{r}$ and $\nabla_{\Omega}$ is the spherical part of $\nabla$ one can write down $\mathbf{L}(i)=-i\left[\mathbf{n}(i) \times \nabla_{\Omega_{i}}\right][22]$.

Thus, after some calculations, one obtains

$$
\mathbf{L}_{m}^{1}(i)=-\sqrt{2} \sum_{p, q} C_{p}^{1} \underset{q}{1} \frac{1}{m} C_{p}^{1}\left(\theta_{i}, \varphi_{i}\right)\left(\nabla_{\Omega_{i}}\right)_{q}^{1}
$$

Using these results for the matrix element of the operator of the nuclear-electronic spin-orbit interaction which determine the transition between two electronic states $K$ and $K^{\prime}$ and the nuclear states $K_{\lambda}$ and $K_{\lambda}^{\prime}$ one obtains

$$
\left\langle\left. K K_{\lambda}\right|^{a} \hat{\mathbf{H}}_{e n}^{L S} \mid K^{\prime} K_{\lambda}^{\prime}\right\rangle=2 \alpha \alpha_{p} g_{\lambda} \sum_{m}(-1)^{m}\left\langle K\left|\sum_{i} r_{i}^{-3} L_{m}^{1}(i)\right| K^{\prime}\right\rangle\left\langle K_{\lambda}\left|I_{-m}^{1}\right| K_{\lambda}^{\prime}\right\rangle
$$




\section{R. L. PAVLOV, P. P. RAYCHEV, M. DIMITROVA-IVANOVICH, C. VALDEMORO}

where the matrix element for the transition of the electronic system has the form:

$$
\begin{aligned}
\left\langle K\left|\sum_{i} r_{i}^{-3} L_{m}^{1}(i)\right| K^{\prime}\right\rangle & =\int_{\mathbf{r}_{1}^{\prime}=\mathbf{r}_{1}} r_{1}^{-3} L_{m}^{1}(i) \rho_{1}\left(K K^{\prime} \mid \mathbf{r}_{1} ; \mathbf{r}_{1}^{\prime}\right) d \mathbf{r}_{1}= \\
& -\sqrt{2} \sum_{p, q} C_{p}^{1} \underset{q}{1} \frac{1}{m} \int_{\mathbf{r}_{1}^{\prime}=\mathbf{r}_{1}} r_{1}^{-3} C_{p}^{1}\left(\theta_{1}, \varphi_{1}\right)\left(\nabla_{\Omega_{1}}\right)_{q}^{1} \rho\left(K K^{\prime} \mid \mathbf{r}_{1} ; \mathbf{r}_{1}^{\prime}\right) d \mathbf{r}_{1},
\end{aligned}
$$

where $\rho\left(K K^{\prime} \mid \mathbf{r}_{1} ; \mathbf{r}_{1}^{\prime}\right)$ is the reduced density matrix of the first rank.

From the general formula of the matrix element of a product of two tensor operators:

$$
\langle K|f \cdot g| K\rangle=\sum_{K^{\prime}}\left\langle K|f| K^{\prime}\right\rangle\left\langle K^{\prime}|g| K\right\rangle
$$

it follows that $\mathbf{R}_{\lambda} \times \mathbf{p}(i)=0$, because the matrix element of $\mathbf{R}_{\lambda}$ differs from zero only when $M_{L}(i)=M_{L}^{\prime}(i)$ while that of $\mathbf{p}(i)$ differs from zero only when $M_{L}^{\prime}(i)=M_{L}(i)+1$ (see [21]). Here as usual $M_{L}(i)$ are the projections of orbital momenta $L(i)$.

In the case of consideration for the matrix elements of operator $\hat{\mathbf{H}}_{e n}^{L S}$, Eq.(33) one obtains

$$
\left\langle K K_{\lambda}^{\prime}\left|\hat{\mathbf{H}}_{e n}^{L S}\right| K^{\prime} K_{\lambda}^{\prime}\right\rangle=2 \alpha \alpha_{p} \sum_{\lambda} g_{\lambda} \sum_{m}(-1)^{m}\left\langle K\left|\sum_{i} r_{\lambda i}^{-3}\left(L_{\lambda}(i)\right)_{m}^{1}\right| K^{\prime}\right\rangle\left\langle K_{\lambda}\left|I_{-m}^{1}(\lambda)\right| K_{\lambda}^{\prime}\right\rangle
$$

where the matrix element of the electronic part take the form, analogous to (39):

$$
\left\langle K\left|\sum_{i} r_{\lambda i}^{-3}\left(L_{\lambda}(i)\right)_{m}^{1}\right| K^{\prime}\right\rangle=-\sqrt{2} \sum_{p, q} C_{p}^{1} \frac{1}{q} \underset{m}{1} \int_{\mathbf{r}_{1}^{\prime}=\mathbf{r}_{1}} r_{\lambda i}^{-3} C_{p}^{1}\left(\theta_{1}, \varphi_{1}\right)\left(\nabla_{\Omega_{1}}\right)_{q}^{1} \rho\left(K K^{\prime} \mid \mathbf{r}_{1} ; \mathbf{r}_{1}^{\prime}\right) d \mathbf{r}_{1}
$$

\section{CONCLUSION}

The results presented here give the possibility to include electron-nuclear spin-spin and contact interactions as well as the interaction between nuclear-spin and electron orbital motion in a density matrix theory for spinpolarized many electron systems in excited and degenerate states. These results allow not only to include the considered relativistic corrections in the variational approach of density matrix methods but also to perform calculations in terms of vector-model and valence-bond schemes.

The matrix elements and expectation values of operators with a complicated analytical form, as for example these of the operator of the electron-nuclear spin-spin interaction given by Eq. (15), are easily reduced to a form which is very simple and suitable for the direct numerical application. The only specific component in the expressions (25), (26), (31) and (32) is contained in the matrix elements of the operators $\hat{\mathbf{H}}_{e n}^{S S}$ and $\hat{\mathbf{H}}_{e n(\text { cont })}^{S S}$. These operators must be constructed and used in the numerical procedure for the normalized spin distribution density, which is the same for a given spin multiplet and does not depend on the considered transition processes be- tween two states $K$ and $K^{\prime}$ of splitted energy levels.

The calculation of the matrix elements of the operator $\hat{\mathbf{H}}_{e n}^{L S}$ (Eqs. (39), (41)) in terms of density matrix of first order is straightforward. The same holds for density functionals built up in terms of density matrices. We could use the local-density functional obtained form of the spin distribution functions or average local-density functional approximation [23] for the spin distribution matrices and, at the end, the first order density matrix in Kohn-Sham equations in order to include the considered relativistic corrections.

In the Barth-Hedin construction [24-26], the most widely used in the Kohn-Sham-type calculations for spin-polarized systems, the energy functional is defined in terms of the first-order density matrix. This does not permit the description of relativistic corrections, which require a two-particle density matrix. In this approach one can only determine the influence of an external magnetic field, and only for the ground state.

The formalism presented here and in $[11,12]$ can be used in the density matrix and density functional methods for the determination of all relativistic corrections described by Breit-Pauli Hamiltonian not only for the ground state, but for any arbitrary state of the spin multiplet as well. The same formalism can be used for the 
calculation of the effect of an external magnetic field.

The use of a suitable minimization procedure, e.g., the local-scaling transformation scheme [27-29], and more precisely speaking, its formulation for spin-polarized sys- tems $[30,31]$, that keeps automatically space and spin symmetry, would allow direct minimization of the energy density-matrix functional, including relativistic terms. This is an aim of our future investigations.
[1] R. McWeeny, Methods of Molecular Quantum Mechanics (Academic Press, New York, 1989).

[2] M. M. Mestetchkin, Mietody priviediennoi matricy plotnosti v kvantovoi moliekuliarnoi tieorii (Methods of Reduced Density Matrices in Quantum Molecular Theory) (Naukova Dumka, Kiev, 1977).

[3] K. Husimi, Proc. Phys. Math. Soc. Jpn 22, 264 (1940).

[4] R. McWeeny, J. Chem. Phys. 42, 1717 (1965).

[5] J. Maruani, Electron and Magnetization Densities in Molecules and Crystals, edited by P. Becker, (Plenum Press, New York, 1980).

[6] V. A. Fock, Zh. Eksp. Teor. Fiz. 10, 961 (1940).

[7] R. McWeeny, Y. Mizuno, Proc. R. Soc., London, Ser. A 259, 554 (1961).

[8] R. McWeeny, Mol. Eng. 7, 7 (1997).

[9] R. McWeeny, Adv. Quantum Chem. 31, 15 (1999).

[10] A. P. Yutsis, I. B. Levinson, V. V. Vanagas, Matiematichieskij formalizm v tieorii uglovogo momienta (Mathematical Formalism of Angular Momentum Theory) (Gospolizdat, Vilnius, 1968).

[11] R. L. Pavlov, A. I. Kuleff, P. Tz. Yotov, J. Maruani, Ya. I. Delchev, in Trends in Quantum System in Chemistry and Physics, edited by J. Maruani et al., (Kluwer Acad. Publishers, Netherlands, 2001), p. 63.

[12] R. L. Pavlov, A. I. Kuleff, P. Tz. Yotov, J. Maruani, Adv. Quantum Chem. 39, 295 (2001).

[13] R. McWeeny, Proc. R. Soc., London, Ser. A 223, 63 (1954).

[14] P.-O. Lowdin, Phys. Rev. 97, 1474 (1955).

[15] C. N. Yang, Rev. Mod. Phys. 34, 694 (1962).

[16] R. McWeeny, Rev. Mod. Phys. 32, 355 (1960); P.-O. Lowdin, Rev. Mod. Phys. 32, 328 (1960); A. J. Coleman, Rev. Mod.Phys. 35, 668 (1963).

[17] H. M. McConnell, J. Chem. Phys. 28, 1188 (1958).
[18] W. H. Moores, R. McWeeny, Proc. R. Soc., London, Ser. A 322, 365 (1973).

[19] A. I. Akhiezer, V. B. Berestetzkii, Kvantovaia Eliektrodinamika (Quantum Electrodynamics) (Nauka, Moskow, 1969).

[20] L. C. Biedenharn, J. D. Louck, Angular Momentum in Quantum Physics (Addison Wesley, Massachusetts, 1981).

[21] I. I. Sobelman, Vviedieniie v tieoriiu atomnykh spiektrov (Introduction to Atomic Spectra Theory), (Nauka, Moscow, 1977).

[22] D. A. Varshalovich, A. N. Moskalev, V. K. Khersonsky, Quantum theory of angular momentum: irreducible tensors, spherical harmonics, vector coupling coefficients, 3nj symbols (World Sci., Singapore, 1988).

[23] O. Gunnarsson, B. I. Lundqvist, Phys. Rev. B 13, 4274 (1976).

[24] U. Barth, L. von Hedin, J. Phys. C 5, 1629 (1972).

[25] M. M. Pant, A. K. Rajagopal, Solid State Commum. 10, 1157 (1972).

[26] A. Rajagopal, J. Gallaway, Phys. Rev. B 7, 1912 (1973).

[27] I. Zh. Petkov, M. V. Stoitsov, Teor. Mat. Fiz. 55, 407 (1983); Yad. Fiz. (Sov. J. Nucl. Phys.) 37, 1167 (1983).

[28] I. Zh. Petkov, M. V. Stoitsov, E. S. Kryachko, Int. J. Quantum Chem. 29, 140 (1986).

[29] I. Zh. Petkov, M. V. Stoitsov, Nuclear Density Functional Theory (Oxford University Press, Oxford, 1991).

[30] R. L. Pavlov, J. Maruani, Ya. I. Delchev, R. McWeeny, Int. J. Quantum Chem. 65, 241 (1997); Erratum, ibid. 68, 75 (1998).

[31] R. L. Pavlov, F. E. Zakhariev, Ya. I. Delchev, J. Maruani, Int. J. Quantum Chem. 65 (1997) 257; Erratum, ibid. 68, 77 (1998).

\title{
НАДТОНКІ ВЗАЕМОДІЇ ЯДЕРНОГО СПІНУ З ЕЛЕКТРОННИМИ СИСТЕМАМИ В МЕТОДАХ МАТРИЩ ГУСТИНИ
}

\author{
Р. Л. Павлов ${ }^{1}$, П. П. Райчев ${ }^{1}$, М. Дімітрова-Івановіч ${ }^{2}$, К. Валдеморо ${ }^{3}$ \\ ${ }^{1}$ Iнститут ядерних дослідженъ та ядерноӥ енергї, \\ Болгарсвка академія наук, Lаріарадске шоссе, 72, \\ BG-1784, Софіл, Болгаріл. \\ ${ }^{2}$ Інститут фізики твердого стану, Болгарсвка академія наук, \\ Царіградске шоссе, 72, BG-1784, Софія, Болгарія. \\ ${ }^{3}$ Інститут математики і фундаментальноӥ фізики, CSIC, \\ 123, Серрано, Мадрид, 28006, Іспані.
}

\footnotetext{
Подано в аналітичній формі матричні елементи та очікувані значення операторів надтонких ядерних взаємодій у багатоелектронних системах. Вони можуть бути застосовані для числових розрахунків у методах матриці густини. Розглянуто електронно-ядерну, спін-спінову та контактну взаємодію а також взаємодію між ядерним спіном та електронними орбітальними рухами. Ці взаємодії, що наявні в ефективному гамільтоніяні Брейта-Паулі, визначають надтонку структуру основних ефектів у спектроскопії, які можна
} 


\section{R. L. PAVLOV, P. P. RAYCHEV, M. DIMITROVA-IVANOVICH, C. VALDEMORO}

спостерігати, наприклад, в експериментах з електронного спінового резонансу (ЕСР) та ядерного магнетного резонансу (ЯМР). На підставі теореми Вігнера-Екарта й використання техніки незвідних тензорних операторів і схеми спін-просторового розділення матричні елементи й очікувані значення цих релятивістських поправок виражено у формі, придатній для прямих числових розрахунків. Остаточний результат записано як добуток (або сума добутків) множників, що визначаються спіном і (або) симетрією кутового моменту кількости руху та просторової частини, яка визначається дією симетризованих тензорних операторів на нормовану матрищю або функцію спінового розподілу чи матрипю зарядового розподілу. Дія цих просторових тензорних операторів однакова для заданої спінової мультиплетности. Шя дія може бути виражена стандартною процедурою, добре означеною в теорії незвідних тензорних операторів. 